\title{
A propos du programme de maturité fédérale en géographie
}

La matière de l'examen de géographie ne peut donner satisfaction. Elle devra être sérieusement amendée, voire repensêe sans tarder. Le Règlement ... de maturité, l) en vigueur depuis 1973 indique les objectifs de l'étude pour les différents types de maturité $A, B, C, D, E$ ainsi que le Programme comprenant cinq domaines principaux. L'énumération laconique de ceux-ci contraste avec la prolixité de la liste des objectifs parue dans Geographica Helvetica (1977, 1 , p. 1-10)2). Cet inventaire ne peut pas donner satisfaction non plus; touffu et désordonné, il ne comprend pas l'énumération systématique, cohérente des notions de base essentielles de notre discipline. Il apparaît plutôt comme un fatras de connaissances et présente des faiblesses évidentes non seulement dans sa rédaction, mais dans sa conception mềme. Un programme structuré devrait réserver une place égale aux différents domaines de la géographie, tout en mettant en évidence, bien sûr, le caractère unitaire et global de celle-ci.

Les commentaires qui suivent, et qui se veulent constructifs, portent exclusivement sur la version française; à dessein, le texte allemand n'a pas été consulté du tout.

Avant de dénoncer les lacunes qui nous paraissent les plus graves, signalons quelques imprécisions fâcheuses. Il ne s'agit pas ici d'ergoter mais, du moment que ce catalogue des objectifs veut fixer la matière jusque dans les détails, ceux-ci doivent être précis et formulés correctement. Il en va de l'esprit même dans lequel l'enseignement sera donné!

Par exemple, peut-on parler des "connaissances de base de la topographie de la Suisse", ou encore d'"apprécier la situation et d'évaluer les distances à l'aide d'exemples concrets"? Que penser de "l'articulation horizontale et verticale des continents"?

Qu'entend-on par:"énumérer les différences de structures par rapport à l'Europe"? S'agit-il de la structure géologique, de la tectonique ? Ou plus loin:"évaluer (à l'aide d'exemples concrets et en fonction de la sécurité face aux catastrophes naturelles, de la position forte ou des possibilités d'échanges commerciaux) les sites géographiques ayant déterminé l'implantation de I'habitat." A la fin du même paragraphe encore (6.2.1.)"Reconnaitre les morphologiques (sic) caractéristiques de la Suisse: verrous, terrasses, cônes de déjection, deltas, etc., et déterminer leur influence sur l'articulation des communautés politiques."

Comme si ces formes mineures étaient toute la Suisse! L'essentiel de ce qui se rapporte à la répartition des altitudes, aux éléments principaux du relief, aux vallées, aux bassins fluviaux et aux réseaux hydrographiques est laissé de côté ici.

Le programme en question, bourré d'expressions de ce genre, comprend sept chapitres, à savoir: 3$)^{2}$

6.2.1. Connaissances de base de la topographie de la Suisse, de I'Europe et des autres (sic) continents.

6.2.2. Géologie de la Suisse.

6.2.3. Méthodes d'observation et de représentation géographiques.

6.2.4.a) Climat, hydrographie, types de sols, végétation.

b) Population, habitat, économie, communications.

c) Action conjuguée du paysage, aménagement du territoire, protection de l'environnement.

A propos du chapitre consacré à la géologie de la Suisse (6.2.2.,p. 6), une question cruciale surgit, à côté de multiples remarques relatives aux notions présentées, aux expressions utilisées. Pour quelles raisons ces rudiments hétéroclites de géologie figurent-ils dans le programme de géographie ? Pourquoi une telle place est-elle accordée à la géologie plutôt qu'à 1 'écologie, à la sociologie, à 1 'ethnologie; ${ }^{4}$ on serait même tenté d'ajouter, avec une pointe d'ironie, à l'astronomie ou au dessin.

La géographie n'est-elle pas suffisamment cohérente et autonome, sa finalité pas assez spécifique pour constituer, à elle seule, la matière d'un examen de maturité fédérale ?

Jean-Pierre Portmann, professeur, Institut de géographie, Coq d'Inde 10, 2000 Neuchâtel 
Les causes de ce rattachement insolite de 1 a géologie à la géographie nous échappent. Fautil les rechercher dans certaines particularités et traditions de l'Ecole suisse alémanique, dans la formation de ses maîtres, etc. ? En tout cas, il tombe sous le sens que les notions géologiques courantes, celles qui constituent le fond commun des connaissances que doit acquérir un bachelier, entrent tout naturellement et logiquement dans la géomorphologie, branche de la géographie physique.

Le chapitre suivant $(6.2 .3 .$, p.6) porte sur les Méthodes d'observation et de représentation géographiques. On s'étonne, en premier lieu, de la position et de l'extension de ce chapitre formulant des recommandations d'ordre didactique. Celles-ci trouveraient logiquement leur place, semble-t-il, dans une introduction. A vrai dire, elles vont même de soi, car elles :sont incluses implicitement dans les autres parties du programme. En fait, elles ne sont pas présentées ici d'une façon systématique, mais énumérées en un chaos de suggestions et de directives.

Plus clair, moins incohérent, nous apparaît le chapitre suivant $(6.2 .4 . a$, p.6), consacré au climat, à l'hydrographie, aux types de sols, et à la végétation. Pourtant le passage consacré à la pédologie est bien restreint et obscur!

Des notions fondamentales de géographie humaine sont présentées dans un autre chapitre (6.2.4. b, p.7) sous le titre Population, habitat, économie, communications. On ne précise pas d'ailleurs s'il s'agit de la Suisse, d'autres pays ou du monde entier. Un des autres sujets laisse aussi planer le doute:'Expliquer les typologies historiques et statistiques des villes et les appliquer à des exemples précis". N'existe-t-il pas d'autres typologies? Se limite-t-on à $1 \mathrm{a}$ Suisse?

Dans un dernier chapitre $(6.2 .5 .$, p.7) on propose 1'étude de quelques applications de la géographie. A notre avis, celles-ci devraient être abordées au gré des différents chapitres, les notions théoriques étant complétées par des exemples d'applications pratiques. Ne serait-ce pas plus indiqué, du point de vue scientifique et didactique, de dénoncer les méfaits de 1 'érosion des sols à propos de l'étude de ceux-ci ? Ou encore de présenter les atteintes à 1'environnement causées par l'industrie en parlant de celle-ci ? D'autres exemples pratiques pourraient être donnés aussi, dans ce cas, quitte à consacrer, in fine, un exposé à la vue d'ensemble des aspects pragmatiques de la géographie.

En guise de conclusion, on se permettra d'insister pour que le Programme, et surtout, le Catalogue des objectifs du Réglement de maturité soient prochainement revus afin de correspondre au contenu réel de la géographie, à ses méthodes et à sa finalité. Tout au long de la formation scolaire, la géographie doit apparâ̂tre comme un ensemble autonome, cohérent de notions essentielles; elle doit être un entraînement à 1 'analyse et à la synthèse. ${ }^{5}$ )

\section{Notes infrapaginales}

1) Règlement des examens fédéraux de maturité (413.12- du 17 décembre 1973-. Etat le ler avri1 1977).

2) Ce catalogue des objectifs ("Lernzielkatalog") a été publié en tant que "Bericht der Spezialkommission des Vereins Schweizerischer Geographielehrer" - VSGg -).

3) Les différences entre les titres de ces chapitres et le programme du Règlement... de maturité (note 1, ci-dessus) sont mineures.

4) VERMOT, Ruth G.: Ethnologie und Schule, Geographica Helvetica, 1979-2 p. 73-76.

5) HENRIET, Jean-Michel.: Curriculum géographique pour adolescents de 16 a 19 ans. Compte-rendu de la Conférence européenne de Londres (29 mars - ler avril 1978). L'Espace géographique 1979/2, p.157. 